\title{
An improved three-coil wireless power link to increase spacing distance and power for magnetic resonant coupling system
}

\author{
Xian Zhang ${ }^{1 *}$, Hao Meng ${ }^{1}$, Bin Wei ${ }^{2}$, Songcen Wang ${ }^{2}$ and Qingxin Yang ${ }^{1}$
}

\begin{abstract}
When wireless power transfer (WPT) technology is used to power the sensor networks, it is necessary to increase the transmission power and distance. We strictly deduced two necessary conditions for strong coupling by coupled mode theory. Next, the transmission power and efficiency as a function of coupling coefficient and quality factor are derived, and the maximum active power of the load depends on the coupling coefficient between resonators, quality factor of the resonator and the drive power. In order to increase transmission power and distance, an improved three-coil wireless power link is proposed. Meanwhile, an experimental platform was established, and the experimental results show that the transmission power can reach $120 \mathrm{~W}$ at a distance of $2.5 \mathrm{~m}$. This proves that the improved three-coil wireless power link is very effective.
\end{abstract}

Keywords: Three-coil wireless power link, Magnetic resonant coupling, Coupled mode theory, Critical strong coupling, Optimal impedance feed

\section{Introduction}

The magnetic resonant coupling wireless power transfer (MRC-WPT) was first proposed by MIT's team in 2007 [1] and is now gaining more spotlights ranging from contactless battery charging of consumer electronics [2], electric vehicles [3-5], to biological implanted devices' power supply $[6,7]$.

WPT technology can be categorized according to their operating frequencies, which are short-range inductive power transmission (IPT) (at the range of a few hundred kilohertz), mid-range MRC-WPT also known as WiTricity (at the range of several $\mathrm{MHz}$ ) and long-range directly microwave radiation (range above $\mathrm{GHz}$ ) $[8,9]$. Different from other two methods, MRC-WPT operates in strong coupling state to transfer energy wirelessly via evanescent non-radiative near field. High-frequency power flows among resonators in a mid-range which is on the order of several times the physical size of transmitting or receiving end when system is tuned into resonant state and finally

\footnotetext{
* Correspondence: 18622169747@163.com

${ }^{1}$ Tianjin Key Laboratory of Advanced Electrical Engineering and Energy

Technology, Tianjin Polytechnic University, Tianjin 300387, China

Full list of author information is available at the end of the article
}

absorbed by the load. Moreover, it interacts weakly with biological tissues or other non-resonant objects.

In the past two decades, IPT is the mainstream of WPT and its transmission distance is short. However, MRC-WPT has a significant increase in transmission distance. For mid-range applications, MRC-WPT is a good choice to reach a range of meters, which is of great significance for the power supply of wireless sensor networks $[10,11]$. Nevertheless, the transmission power of MRC-WPT system decreases with increasing transmission distance because the magnetic coupling is relatively small at longer distances. Therefore, power transmission range needs to be extended to achieve more flexibility.

Several methods were previously proposed to accommodate power pickups when there is deviation in radial direction. In [12], a multiphase system is proposed to obtain a wider power delivery zone. In [13], a wide power transmission area is achieved by two transmitters. In [14], a cooperative operating coupler featuring tight-strong coupling is proposed to enhance anti-offset capability. However, transmission distance cannot be extended in coil axis directions by these methods.

In [15-17], superconductor is utilized to reduce the coil losses and increase transmission distance. But it is 
very expensive and hard to popularize at the moment. In [18], the transmission distance is adjusted by changing the driving frequency of power source or controlling the loading effect of transmitting coil and receiving coil. However, the range adaptation is only intended to improve the performance at short distances where resonant frequency splitting occurs. The maximum transmission distance after the critical coupling point is still limited by the vanishing magnetic coupling.

In this paper, we provide an improved three-coil wireless power link to maximize the transmission distance and power at a reasonable level of efficiency. First, the time domain solution of power exchange between two resonators is achieved through coupled mode theory. Two key prerequisites of strong coupling are deduced by discussing the angular frequency, mode coupling factor and quality factor. After that, the overall transmission efficiency and the maximum power of the load are analysed. Finally, a three-coil wireless power link containing two helix resonators and a device coil was established, and power on the load reached $120 \mathrm{~W}$ with transmission distance up to $2.5 \mathrm{~m}$. Measured values have an error of $\pm 2.5 \%$ compared to theoretical ones.

\section{Methods/experimental section}

It is necessary to increase the load power and to extend the transmission distance in axial direction for MRC-WPT system. Though there is high power over kilowatts and long distance over several kilometres via micro-wave transmission, it is far from safety and stability compared to MRC-WPT by using the non-radiative near field. When the electrical parameters are fixed, the maximum active power the load can get depends on the coupling coefficient between resonators, quality factor of the resonator and the drive power. Therefore, if we intend to increase the load power, the overall contribution of three parameters above should be concerned.

It can be seen from the calculation formula $(Q=\omega L / R)$ of the resonator quality factor that the quality factor can be improved in two aspects: (1) increasing the ratio of inductance and resistance and (2) increasing the natural resonant frequency. In this paper, in order to increase the quality factor of the resonators, the oxygen-free copper tube with smooth surface is relatively nice material, because it has good conductive property and skin effect is reduced to some extent. It should be noted that there is only limited improvement for the ratio of inductance and resistance. The efficient way to get a larger quality factor is to increase the natural resonant frequency. With participation of the distributed capacitance between coil tubes, the resonant frequency is up to $13.56 \mathrm{MHz}$ with the quality factor of 1100 1500.

In our project, high-frequency power is fed directly to the transmitting resonator. The high-frequency signal is generated by DDS and amplified by MOS transistor M1 and M2, as shown in Fig. 1a. C1 and C2 are used to adjust dead time; D1 and D2 are responsible for freewheeling; C3 C5 and L1 are responsible for impedance matching. Figure 1b shows the way of feed; Fig. 1c shows the dashboard of power amplifier. Detailed parameters of improved three-coil wireless power link system are shown in Table 1.

\section{Characteristics of MRC-WPT by coupled mode theory}

A general configuration of MRC-WPT system is shown in Fig. 2; there are three sections to fulfil the functions of power coupling, transformation and transmission. Power on resonator $a_{1,2}$ is acquired through inductive coupling to achieve the conversion of low voltage/high current and high voltage/low current between resonators and source or device coil. Once the system is tuned into resonant state, power exchanges between the resonators through EM resonantly coupling which results in a relatively low transmission loss by high impedance performance. $V_{a 1,2}, I_{a 1,2}, C_{a 1,2}$ and $R_{a 1,2}$ are equivalent to voltage, current, capacitance and resistance of resonator $a_{1,2}$, respectively; $I_{1,2}, R_{S, D}$ and $L_{S, D}$ are current, resistance and induction of source coil and device coil; $V_{S}$ is driving voltage; $R_{L}$ is load resistance; $M_{S 1}, M_{1,2}$ and $M_{2 D}$ are mutual induction between the coils. Here, the coupling effect between non-resonant source and device coil is ignored.

\subsection{Prerequisites for efficient wireless power transfer}

The coupled mode theory is usually employed to describe the general laws of wave interaction phenomena such as optical waves and sound waves. It is very suitable for modelling the resonant energy exchange. For the lossless resonators in a MRC-WPT system, energy exchange between resonators can be described by two decoupled differential equations as (1) [19].

$$
\left\{\begin{array}{l}
\frac{d a_{1}}{d t}=j \omega_{1} a_{1}+\kappa_{12} a_{2} \\
\frac{d a_{2}}{d t}=\kappa_{21} a_{1}+\mathrm{j} \omega_{2} a_{2}
\end{array}\right.
$$

where the forward rotation mode of resonators is denoted as $a_{1}$ and $a_{2}$ whose second norm has energy dimension; $\omega_{1,2}$ are natural resonant angular frequency; $\kappa_{12}$ and $\kappa_{21}$ are mode coupling factors; and there is a relationship with coupling coefficient $k$ which is $\kappa=\omega k / 2$. For passive lossless system, energy exchange occurs between modes and meets conservation for overall energy. Any change of oscillation mode needs to go through natural resonant periods under weak coupling, so it comes to $a_{1}(t)=\boldsymbol{A}(t) * \exp$. $\left[0.5 \mathrm{j}\left(\omega_{1}-\omega_{2}\right) t\right]$, where $\boldsymbol{A}(t)$ is a slowly varying time domain function. Assume there is an 


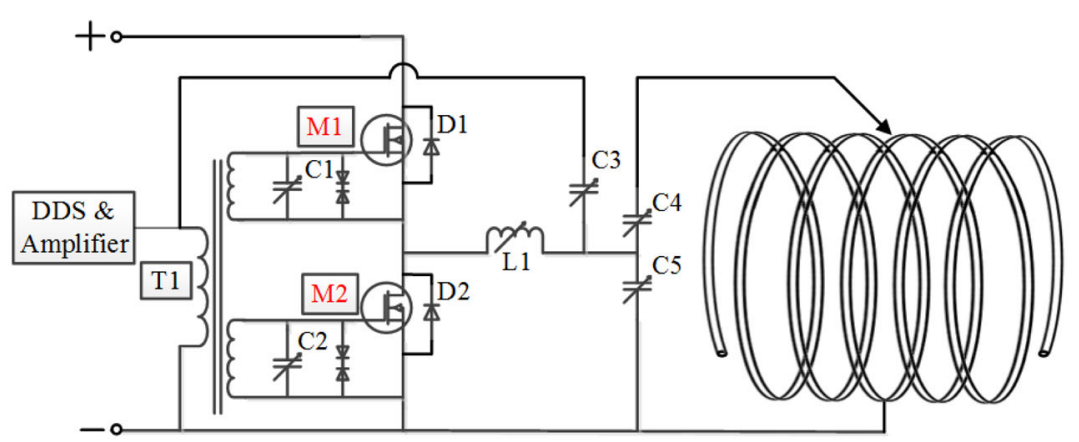

(a) The structure diagram of three-coil power link

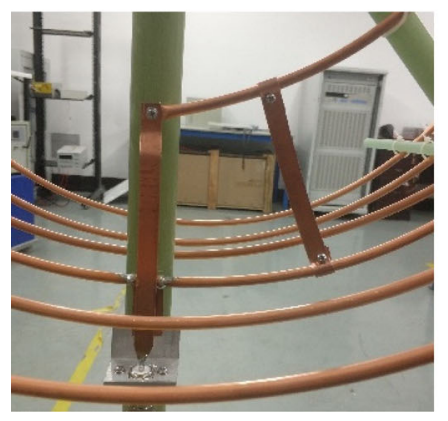

(b) The way of feed

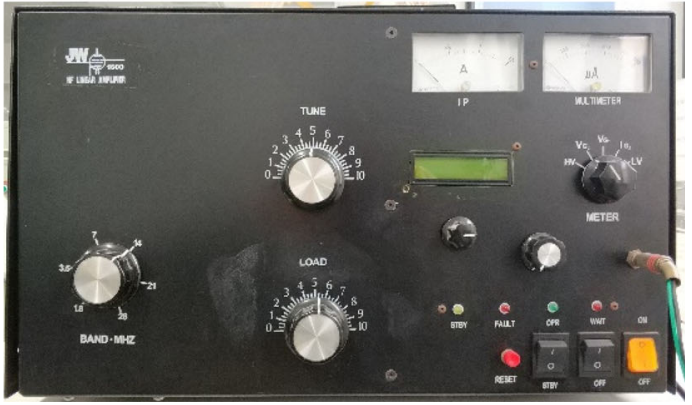

(c) The dashboard of power amplifier

Fig. 1 Structure diagram of three-coil power link and experimental system. a The working principle of the three-coil wireless charging system. The waveform is generated through the DDS circuit. After multilevel amplification, the power is loaded onto the resonant coil. $\mathbf{b}$ The way of power loading, which improves the power conversion efficiency by the way of central feeding. The power supply prototype used in the experiment is shown in $\mathbf{c}$. a The structure diagram of three-coil power link. $\mathbf{b}$ The way of feed. $\mathbf{c}$ The dashboard of power amplifier

initial condition as $\Gamma\left\{t=0, a_{1}(t)=\boldsymbol{A}(t) ; a_{1}(t)=0\right\}$, the solution can be derived as (2), where $\omega$ is the average value of $\omega_{1}$ and $\omega_{2}$.

$$
\left\{\begin{array}{l}
a_{1}(t)=\mathrm{A}(0)\left[\cos (\Omega t)+j \frac{\omega_{1}-\omega_{2}}{2 \Omega} \sin (\Omega t)\right] \cdot e^{\mathrm{j} \bar{\omega} t} \\
a_{2}(t)=\mathrm{A}(0) \kappa_{21} \sin (\Omega t) / \Omega \cdot e^{j \omega t}
\end{array}\right.
$$

Their two eigenvalues are $\lambda_{12}= \pm \Omega=\left[\left(\omega_{1}-\omega_{2}\right)^{2} / 4\right.$ $\left.-\kappa_{12}{ }^{*} \kappa_{21}\right]^{0.5}$. There is the first prerequisite that the maximum energy exchange exists unless $\omega_{1}=\omega_{2}$. It is simple and intuitive but explains why the two circuits have to be tuned into resonance.

For a lossy coupling system, the natural resonant angular frequency will have an offset, and modified resonant angular frequency can be expressed as $\omega^{\prime} 1,2$ $=\omega_{1,2}\left(1+j / 2 Q_{1,2}\right)$. The eigenvalues can be expressed as (3) by Taylor expansion, when the quality factor $Q$ is high enough. In general, for more convenient impedance matching, the resonators are designed to be the same size, resonant frequency and quality factor, so there are $Q_{1}=Q_{2}=Q$ and $\omega_{1}=\omega_{2}=\omega$.

$$
\lambda_{1,2}=-\left(\frac{\omega}{Q}\right) \pm \sqrt{\kappa_{12} \kappa_{21}}
$$

If we intend to obtain a sustaining growth in oscillation, it must meet the condition that there is a positive root and the item within the radical sign is a real number. In general, the parameters of resonators are designed to be the same, so there are $Q_{1}=Q_{2}=Q$ and $\omega_{1}=\omega_{2}=\omega$. And the second prerequisite is shown as (4) after simplification.

$$
\left|\kappa_{12} \kappa_{21}\right|>>\frac{\omega^{2}}{4 Q^{2}}
$$

The two mode coupling factors denote the energy coupling rate; the two quality factors denote the loss rate. In other words, the resonators with high-quality factors are needed to form a low-loss bridge for energy flowing between the source and device.

Energy exchange relationship between resonators in normalization is shown as Fig. 3 in which $\left|a_{1}\right|^{2},\left|a_{2}\right|^{2}$ and $\left|a_{1}+a_{2}\right|^{2}$ indicate energy of resonator 1,2 and overall system. Presence of quality factor $Q$ makes the overall energy decays exponentially. Reason of attenuation in linear system includes Joule loss and radiation 
Table 1 Parameters of improved three-coil wireless power link system

\begin{tabular}{lll}
\hline Parameters & Resonators & Load coil \\
\hline Materials & Copper pipe & Silver-plating copper wire \\
Conductivity $\left(10^{7} \mathrm{~S} / \mathrm{m}\right)$ & $5.56 \sim 6$ & 6.06 \\
Number of turns & 4.25 & 4 \\
Radius of wire $(\mathrm{mm})$ & 3 & 2.5 \\
Radius of the coil $(\mathrm{cm})$ & 30 & 10 \\
Conductor spacing $(\mathrm{cm})$ & $6 \pm 2.5$ & - \\
DC resistance $(\Omega)$ & 0.046 & 0.002 \\
Resonant frequency $(\mathrm{MHz})$ & $10.1-14.5$ & - \\
Quality factor & $1000 \sim 1500$ & - \\
\hline
\end{tabular}

loss which can be expressed as absorption and radiation of $Q$ value. As shown in Fig. 3a with $\omega_{1}=\omega_{2}$ and different mode coupling factor, though there is almost 100\% energy exchange, it cannot keep sustaining and attenuating through several cycles. Usually, the distance between the two resonators is several times of them. So it can be called as loose coupling mode. In Fig. 3b, when angular frequencies differ greatly, most of energy were stored in resonator 1 , and the receiver gets very limited energy which is the main reason of decline in transmission power and overall efficiency, which can be called as weak coupling mode. As soon as the two requirements are met, it is working under the strong coupling state in which proportion of energy exchange reaches 100\% theoretically, as shown in Fig. 3c, which can be called as strong and tight coupling mode.

\subsection{Transmission performance for MRC-WPT system}

For the system as shown in Fig. 2, it is assumed that energy between resonators is exchanged only through $M_{12}$, and coupling coefficients $k_{i j}$ are defined as $k_{i j}=$ $M_{i j} /\left(L_{i} L_{j}\right)^{1 / 2}\left(i=S, 1,2 ; j=1,2\right.$, D). If $k_{2 D}$ is small enough, equivalent quality factor $Q^{\prime}$ of receiver can be expressed as:

$$
Q^{\prime}=\frac{Q Q_{L}}{Q+Q_{L}}
$$

Quality factor reflects the ratio of EM energy stored in circuit and energy consumed in a cycle when in resonance. Energy consumption comes from the resonator itself and load resistance. For high- $Q$ resonator, $R_{D}$ is small enough to be ignored, and the value of equivalent quality factor $Q^{\prime}$ is more close to $Q_{L}$. Therefore, active power on the load $P_{L}$ is

$$
P_{L}=\frac{A^{2}}{\omega_{0}} \cdot \frac{4 k_{12}^{2}}{Q_{L}} \cdot\left[k_{12}^{2}+1 /\left(Q Q_{L}\right)\right]^{-2}
$$

So when resonance occurs, $P_{L}$ is a multivariate function of excitation power, resonant angular frequency, coupling coefficient and quality factor. Figure 4 shows the variation of normalized $P_{L}$ with load quality factor and coupling coefficient, wherein logarithmic coordinates are used to facilitate observation except $z$-axis. When operating frequency is kept constant, $P_{L}$ is not always increasing with $k_{12}$, but reaches maximum under a certain condition which is the critical coupling position. In general, coupling coefficient changes with the spacing variation, and other parameters are fixed

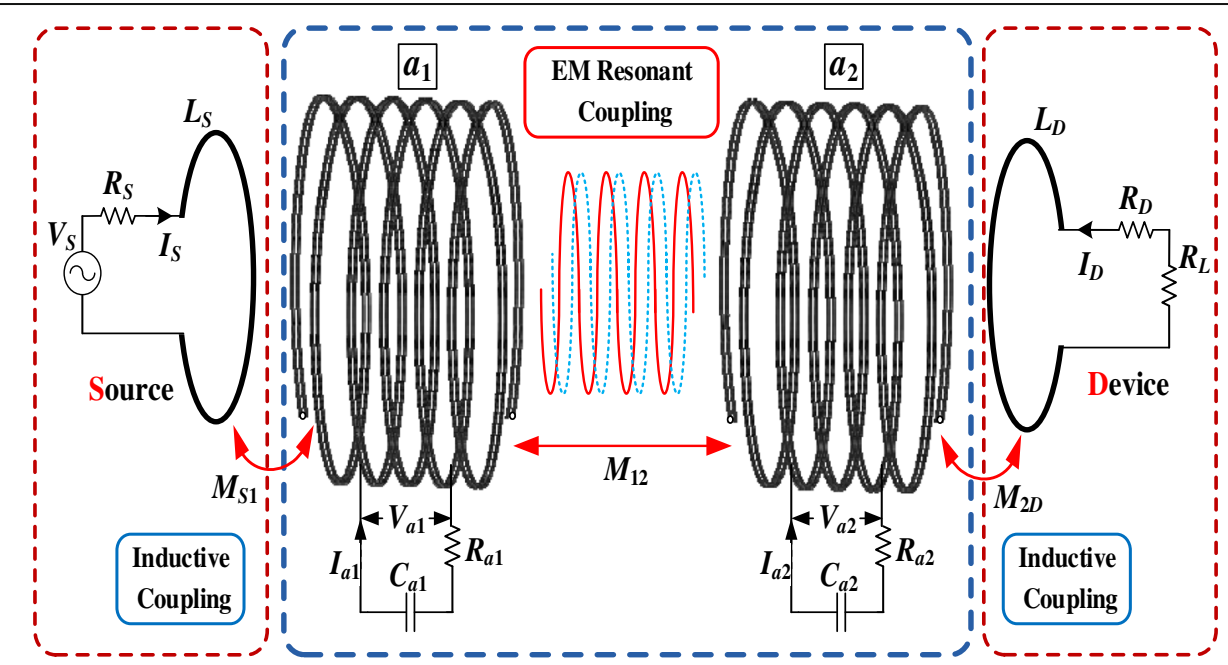

Fig. 2 Directly field-circuit model of a MRC-WPT system. The analysis of the coupling principle of the field-circuit is shown in Fig. 2. The source coil and device coil are coupled with the resonant coils through inductive coupling, and the resonant coils perform power transmission through EM resonant coupling 


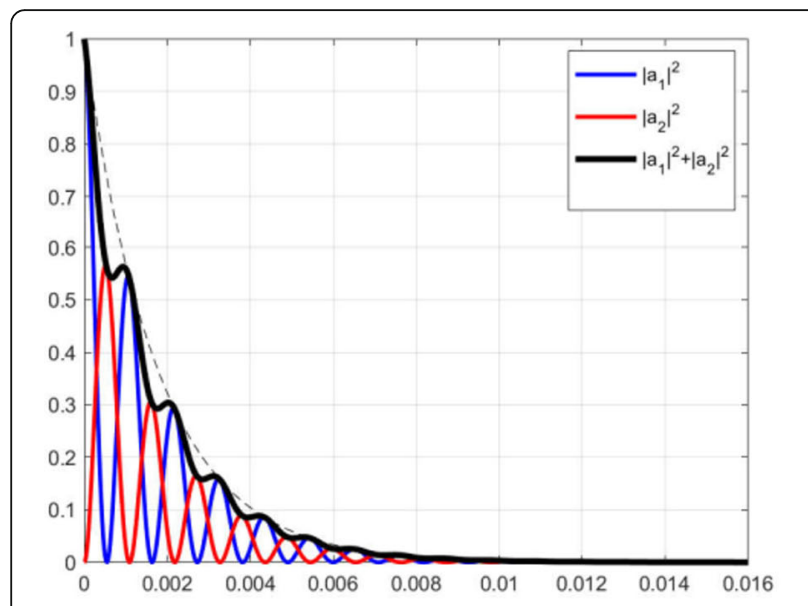

(a) loose coupling mode

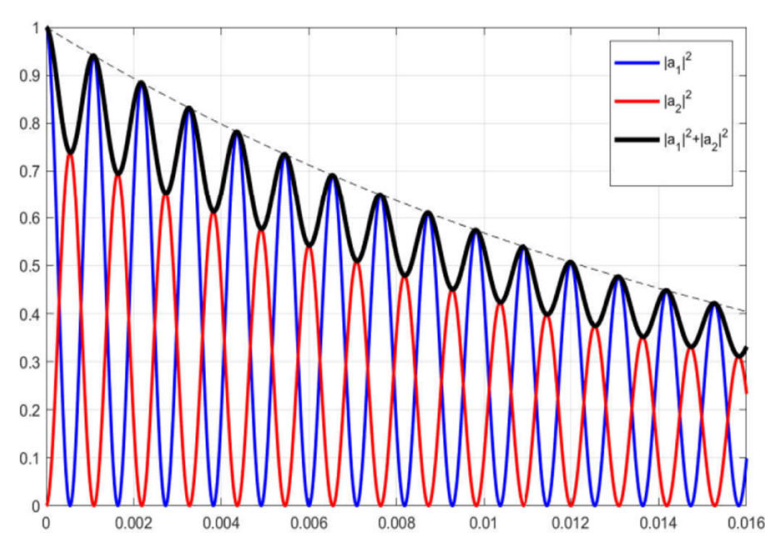

(b) weak coupling mode

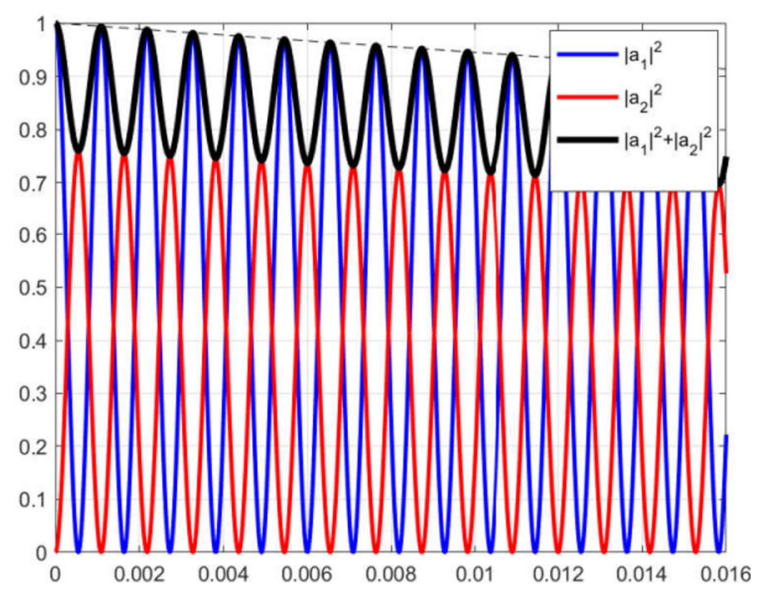

(c) strong and tight coupling mode

Fig. 3 Schematic of normalized energy exchanging. Normalized energy exchanging is solved by coupled mode method. In loose coupling mode, the power of the system quickly attenuates to zero. In weak coupling mode, the rate of energy exchange between the transmitter and the receiving end is very small; it means that the transmitting efficiency is low. In strong and tight coupling mode, the system will work in a highly efficient transmission and low-loss state. a Loose coupling mode. $\mathbf{b}$ Weak coupling mode. $\mathbf{c}$ Strong and tight coupling mode

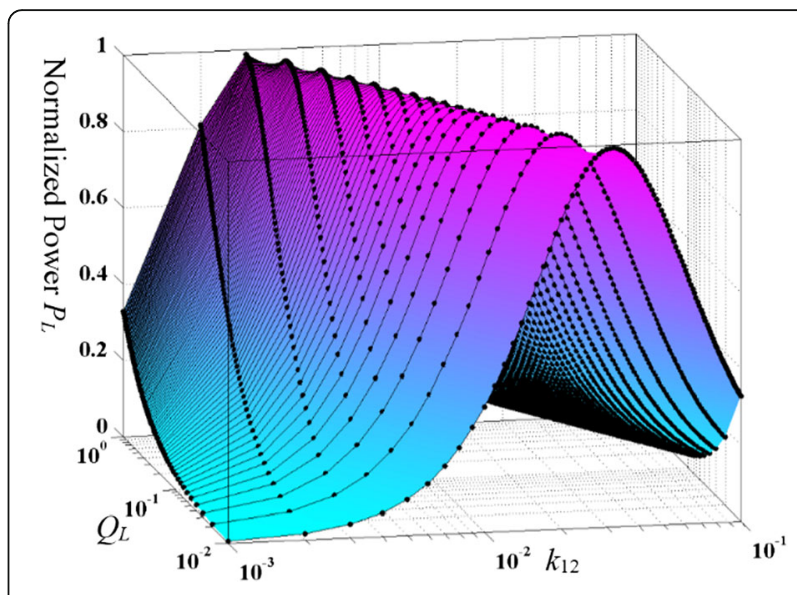

Fig. 4 Normalized active power $P_{L}$ as a function of $k_{12}$ and $Q_{L}$. LEGEND: The abscissa axis is the coupling coefficient $k_{12}$ and the load quality factor $Q_{L}$. They are two important parameters affecting the power. The normalized active power $P_{L}$ is displayed on the ordinate axis. It is a unimodal function of the coupling coefficient and the quality factor. Only when both of them get the appropriate value, can the power reach the maximum

values. Thus, the maximum active power obtained by the load is

$$
P_{L \max }=\frac{A^{2}}{\omega_{0}} \cdot Q
$$

To derive the transmission efficiency function, we assumed that external objects do not participate in energy exchange. Active power consumption result in the loss on load power, $a_{1}$ and $a_{2}$. While power consumption can be divided into Joule losses and radiation losses of a certain dose, both can be embodied by equivalent quality factor. So the overall efficiency $\eta$ can be expressed as:

$$
\begin{aligned}
\eta & =\frac{P_{L}}{P_{\text {total }}} \\
& =\frac{\left(Q / Q_{L}\right) \cdot\left(k_{12} Q\right)^{2}}{\left(1+Q / Q_{L}\right)^{2}+\left(1+Q / Q_{L}\right) \cdot\left(k_{12} Q\right)^{2}}
\end{aligned}
$$

And the maximum transmission efficiency can be obtained from (8) as:

$$
\eta_{\max }=1-2 /\left(1+Q / Q_{L}\right)
$$

It can be drawn that linear resonant coupling system transmission efficiency depends mainly on two factors. The first item $Q / Q_{L}$ shows the role of impedance matching circuit. Here, the load quality factor is generally less than the oscillator quality factor which ensures that transmission efficiency is always over zero. When $Q_{L}<<Q$, system efficiency approaches the maximum which indicates power is all absorbed by the load. When $Q_{\mathrm{L}}=Q$, efficiency is almost zero, indicating that power stored around the load 
in reactive form. The second item $k_{12} Q$ shows the changes of two resonators' space distance. When distance increases, coupling coefficient is reduced with efficiency decreased. When distance becomes smaller, $k_{12} Q$ increases and the efficiency increases accordingly. The specific variations between efficiency and the two factors are shown in Fig. 5, while efficiency curve of critical state when satisfying (9) and $k_{12} Q=1$ are drawn at the same time.

\section{Improved three-coil wireless power link}

The coupling mechanism of MRC-WPT system is often composed of four coils which are source coil, two resonators and device coil, as shown in Fig. 2. In section III, the energy exchange between two resonators is mainly analysed by the coupled mode theory. There is also energy transmission between source coil or device coil and resonators. In the resonant state, the transmission efficiencies between two adjacent coils can be expressed by circuit theory as:

$$
\left\{\begin{array}{l}
\eta_{S 1}=\frac{P_{1}}{P_{S}}=\frac{\left(1+k_{2 D}^{2} Q Q_{D}\right) k_{S 1}^{2} Q_{S} Q}{\left(1+k_{S 1}^{2} Q_{S} Q\right)\left(1+k_{2 D}^{2} Q Q_{D}\right)+k_{12}^{2} Q^{2}} \\
\eta_{12}=\frac{P_{2}}{P_{1}}=\frac{k_{12}^{2} Q^{2}}{1+k_{12}^{2} Q^{2}+k_{2 D}^{2} Q Q_{D}} \\
\eta_{2 D}=\frac{P_{L}}{P_{2}}=\frac{k_{2 D}^{2} Q Q_{D}}{1+k_{2 D}^{2} Q Q_{D}} \cdot \frac{R_{L}}{\left(R_{L}+R_{D}\right)}
\end{array}\right.
$$

where $Q_{\mathrm{S}}=\omega_{0} L_{\mathrm{S}} /\left(R_{\mathrm{S}}+R_{\mathrm{S}}{ }^{\prime}\right), R_{\mathrm{S}}{ }^{\prime}$ is internal resistance of power source, $Q_{\mathrm{D}}=\omega_{0} L_{\mathrm{D}} /\left(R_{\mathrm{D}}+R_{\mathrm{L}}\right)$. From $\eta_{\mathrm{S} 1}$ and $\eta_{2 \mathrm{D}}$, it can be seen that when the quality factor of coils and $k_{12}$ are constant, the increase of coupling coefficients $k_{\mathrm{S} 1}$ and $k_{2 \mathrm{D}}$ contribute to the improvement of the efficiency. However, the source coil and the device coil have the function

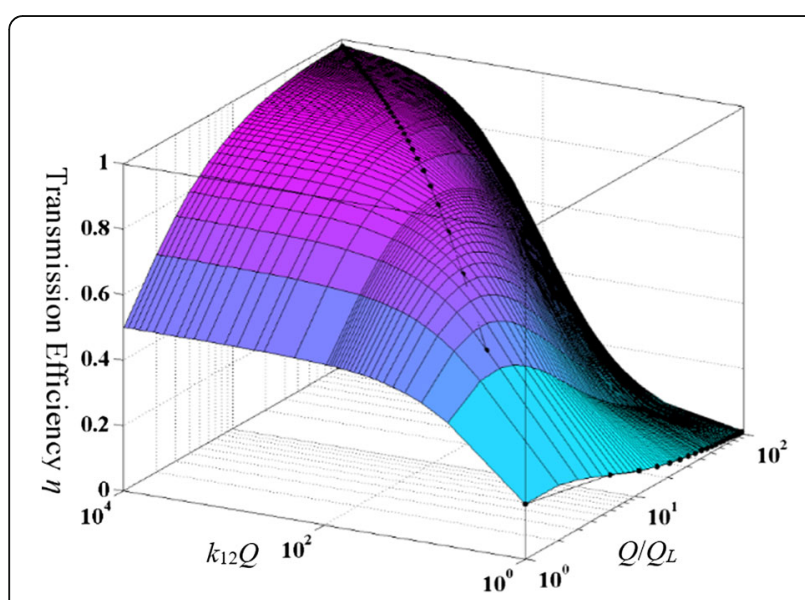

Fig. 5 Transmission efficiency $\eta$ as a function of $Q / Q_{L}$ and $k_{12} Q$. The abscissa axis is $Q / Q_{L}$ and $k_{12} Q$. For system efficiency, it is related to many factors. In this paper, we combine the relevant factors to see the relationship of efficiency more directly of impedance matching which is achieved by adjusting the distance between the source coil or device coil and the resonators. Two resonators are essential in mid-range applications, and in order to reduce the transmission loss between the coils, electrical energy can be directly fed to the resonator so that the source coil can be omitted. Therefore, an improved three-coil wireless power link is proposed, which consists of a transmitting resonator, a receiving resonator and a device coil. The transmitting resonator and the receiving resonator are symmetrical.

For the coupling coefficient $k_{12}$ between the two resonators, it determines the state of system. Once the coupling coefficient $k_{12}$ exceeds the threshold value, frequency splitting disappears and $P_{L}$ decreases with the reduction of $k_{12}$. In general, MRC-WPT system is intended to operate in a far enough distance to increase the flexibility of power supply. Therefore, the remaining method to reduce damping effect is to choose the coils with larger dimension and more number of turns. However, for helical resonator, this is also a way to reduce the flexibility of MRC-WPT.

Only the characteristic impedance of the coil matches the output impedance of the power supply; there will be no reflected power. The degree of matching is commonly measured by the reflectance coefficient, as shown in (11).

$$
\Gamma=\frac{Z_{\mathrm{o}}-R_{S}^{\prime}}{Z_{\mathrm{o}}+R_{S}^{\prime}}
$$

where $Z_{\mathrm{o}}$ is the characteristic impedance of the resonator. In order to achieve this purpose, the resonators adopt $\gamma$ matching method whose feed position is in the centre of the coil, and its characteristic impedance can be adjusted until it matches the output impedance of the

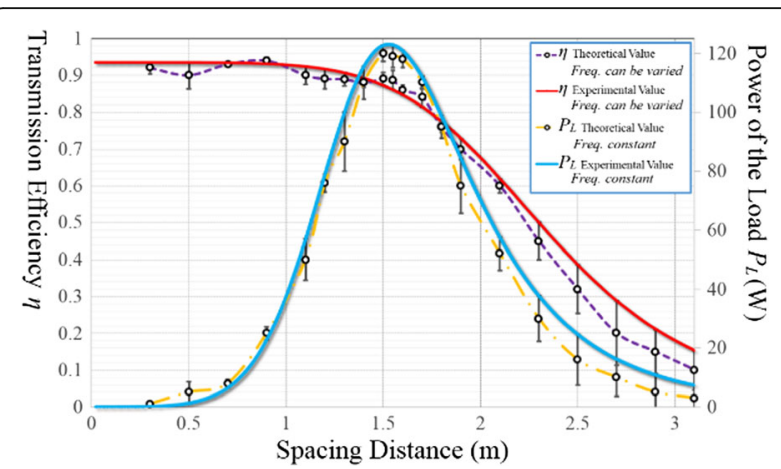

Fig. 6 Transmission efficiency $\eta$ and power of the load $P_{L}$ as a function of spacing distance. Transmission efficiency $\eta$ and power of the load $P_{L}$ as a function of spacing distance is shown in this figure. The transmission efficiency $\eta$ curves of theoretical value and experimental value when the frequency can be varied decay with the increase of distance. When the frequency is constant, there is a peak value of the load $P_{L}$. The curve shows that the error between theoretical calculation and actual measurement is relatively small 


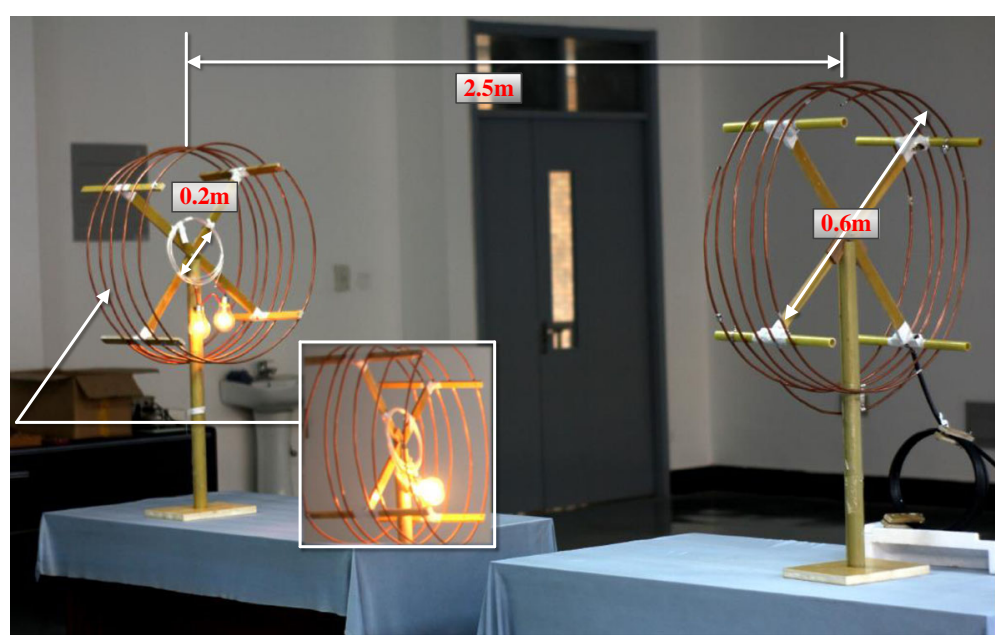

Fig. 7 Wireless power transfer to two 60-W bulbs under interval of $2.5 \mathrm{~m}$. The distance between the transmitting coil and the receiving coil is $2.5 \mathrm{~m}$. The receiving end power through the coupling of up to $120 \mathrm{~W}$ and successfully lit the two lights

power supply. The output impedance of the power supply is generally $50 \Omega$. Meanwhile, the resonator of reception side uses the same method to adjust impedance. In this way, the maximum source power is loaded to resonator and efficiently transmitted to the load.

In order to verify the transmission performance of the improved three-coil link, a three-coil wireless power transfer system was established and its transmission performance was tested within the distance of 0 to $3 \mathrm{~m}$. Theoretical and experimental values of $\eta$ and $P_{L}$ as a function of spacing distance is shown in Fig. 6. Two $60-\mathrm{W}$ bulbs connected in parallel are the load whose power has experienced a process of first increasing and then decreasing. And the power reaches a maximum of $120 \mathrm{~W}$ at a distance of $1.5 \mathrm{~m}$. It is the critical coupling point when frequency keeps constant. The efficiency curve shows that the closer two resonators, the higher the efficiency is when frequency can be varied. However, it will decrease when distance is greater than $1.5 \mathrm{~m}$. When source power is increased to $300 \mathrm{~W}$, it can still supply $120 \mathrm{~W}$ at $2.5 \mathrm{~m}$ under efficiency about $32 \%$ from DC power to the load, as shown in Fig. 7.

\section{Conclusions}

Precondition of energy exchange in strong coupling can be expressed as follows: the angular frequency of resonators is equal, and the product of coupling coefficient and quality factor is much larger than 1 . We further derived that efficiency was constrained by $Q / Q_{L}$ and $k_{12} Q$ which reflect the contribution by electrical structure and spacing distance respectively. The maximum active power of the load depends on the coupling coefficient between resonators, quality factor of the resonator and the drive power. An improved three-coil wireless power link is proposed to maximize the transmission distance and power at a reasonable level of efficiency. Meanwhile, a centre feed method is applied to resonators to achieve good impedance matching. In order to verify the theory, two $220 \mathrm{~V} / 60 \mathrm{~W}$ bulbs are used as load, and MRC-WPT system with helical centre feed coils are designed. Experiments show that $120 \mathrm{~W}$ can be wirelessly transmitted at a distance of $2.5 \mathrm{~m}$ which is two times the power, 1.25 times the distance and 2.13 times the efficiency of [1]. In the future work, we can use this system to supply power for wireless sensor networks.

\section{Abbreviation}

MRC-WPT: Magnetic resonant coupling wireless power transfer; WPT: Wireless power transfer

\section{Acknowledgements}

This work was supported by the Science and Technology Project of State Grid Corporation of China: Research on Electromagnetic Safety Evaluation and Test method of Wireless Charging for Electric Vehicles.

\section{Authors' contributions}

$X Z$ is the main writer of this paper. He proposed the main idea, deduced the performance of WPT detection, completed the simulation and analysed the result. HM introduced the algebraic equation of power and efficiency by coupled mode theory. BW and SW provided experimental environment and completed the experiment. QY gave some important suggestions for threecoil wireless power link. All authors read and approved the final manuscript.

\section{Authors' information}

Zhang Xian, male, Ph.D., Associate professor of Tianjin Key Laboratory of Advanced Electrical Engineering and Energy Technology come from Tianjin Polytechnic University (TJPU), Tianjin, China, was born in Hebei, China, on Dec 1983. He received the Bachelor degree from Hebei University of Technology (HEBUT) in 2009, majoring in Electrical Engineering, and the Doctor Degree in Engineering form HEBUT in 2012. Now, he majors in wireless power transmission and standards in TJPU.

Meng Hao was born in Hebei Province, People's Republic of China, in Apr 1990. He received B.S. degree from Hebei University in 2016, majoring in Electrical Engineering. He is currently working toward the M.S. degree in School of Electrical Engineering and Automation, Tianjin Polytechnic University. His research interest is wireless power transfer. Wei Bin, male, Ph.D., Professor of engineering, come from China Electric Power Research Institute (CEPRI), Beijing, China, was born in Hubei, China, on 
May 1978. He received the Bachelor degree from Changsha University of Science and Technology in 2002, majoring in Power System and Automation, and the Doctor Degree in Engineering from Huazhong University of Science and Technology (HUST) in 2007. Now, he majors in in electric vehicle wireless charging technology and standards in CEPRI. Wang Songcen, male, Ph.D., Senior engineer, comes from China Electric Power Research Institute (CEPRI), Beijing, China, was born in Hernan, China, on Dec 1979. He received the Bachelor degree from North China Electric Power University in 2001, majoring in Automation, and the Master and Doctor Degree in Power Electronic from CEPRI in 2010. Now, he works on wireless power transfer and energy storage in CEPRI.

Yang Qingxin, male, Ph.D., Professor of Tianjin Key Laboratory of Advanced Electrical Engineering and Energy Technology come from Tianjin Polytechnic University (TJPU), Tianjin, China, was born in Hebei, China, on Apr 1961. He received the Bachelor degree from Hebei University of Technology (HEBUT) in 1986, majoring in Electrical Engineering, and the Doctor Degree in Engineering form HEBUT in 1997. Now, he majors in Engineering electromagnetic field and magnetic technology in TJPU.

\section{Competing interests}

The authors declare that they have no competing interests.

\section{Publisher's Note}

Springer Nature remains neutral with regard to jurisdictional claims in published maps and institutional affiliations.

\section{Author details}

${ }^{1}$ Tianjin Key Laboratory of Advanced Electrical Engineering and Energy Technology, Tianjin Polytechnic University, Tianjin 300387, China. ${ }^{2}$ China Electric Power Research Institute, Beijing 100085, China.

Received: 14 February 2018 Accepted: 10 May 2018

Published online: 25 May 2018

\section{References}

1. A Kurs, A Karalis, R Moffatt, et al., Wireless power transfer via strongly coupled magnetic resonances. Science 317(5834), 83-86 (2007)

2. VT Nguyen, SH Kang, JH Choi, et al., Magnetic resonance wireless power transfer using three-coil system with single planar receiver for laptop applications. IEEE Trans. Consum. Electron. 61(2), 160-166 (2015)

3. X Zhang, Z Yuan, Q Yang, et al., Coil design and efficiency analysis for dynamic wireless charging system for electric vehicles. IEEE Trans. Magn. 52(7), 1-4 (2016)

4. SC Moon, GW Moon, Wireless power transfer system with an asymmetric four-coil resonator for electric vehicle battery chargers. IEEE Trans. Power Electron. 31(10), 6844-6854 (2016)

5. X Zhang, P Zhang, Q Yang, et al., Magnetic shielding design and analysis for wireless charging coupler of electric vehicles based on finite element method. Transactions of China Electrotechnical Society 31(1), 71-79 (2016)

6. YY Ko, SL Ho, WN Fu, et al., A novel hybrid resonator for wireless power delivery in bio-implantable devices. IEEE Trans. Magn. 48(11), 4518-4521 (2012)

7. D Ahn, M Ghovanloo, Optimal design of wireless power transmission links for millimeter-sized biomedical implants. IEEE Transactions on Biomedical Circuits and Systems 10(1), 125-137 (2016)

8. Q Yang, P Zhang, L Zhu, et al., Key fundamental problems and technical bottlenecks of the wireless power transmission technology. Transactions of China Electrotechnical Society 30(5), 1-8 (2015)

9. X Zhang, Z Yuan, P Zhang, et al., Transmitting capacity estimation and verification for wireless power transmission system via electromagnetic resonant coupling. Transactions of China Electrotechnical Society 30(19), 47-54 (2015)

10. $Y$ Dong, $L Y$ Y, $\mathrm{L} L \mathrm{Li}$, et al., Resonance wireless power transmission technology theory and its applications in the micro-sensor. Microcomputer \& Its Applications 33(3), 1-4 (2014)

11. B Kallel, O Kanoun, H Trabelsi, Large air gap misalignment tolerable multi-coil inductive power transfer for wireless sensors. IET Power Electron. 9(8), 1768-1774 (2016)

12. GA Covic, JT Boys, M Kissin, et al., A three-phase inductive power transfer system for roadway-powered vehicles. IEEE Trans. Ind. Electron. 54(6), 3370-3378 (2007)
13. JS Raymond, J Raymond, Long range inductive power transfer with superconducting oscillators. Ann. Phys. 325(2), 287-299 (2010)

14. X Zhang, $Y$ Jin, Z Yuan, et al., Analysis of tight-strong coupling mode for dynamic wireless charging of electric vehicle. Automation of Electric Power Systems 41(2), 79-83 (2017)

15. X Nie, Y Wang, X Wang, et al., Analysis of efficiency of wireless power transfer system via strongly coupled magnetic resonance with superconducting resonance coil. Chinese Journal of Low Temperature Physics 39(2), 32-37 (2017)

16. G Zhang, H Yu, G Liu, et al., Superconducting wireless power transfer technology. Southern Power System Technology 9(12), 3-10 (2015)

17. C Zhao, G Zhang, Z Liu, The research of the receiving experiment of a magnetic coupling resonant wireless power transmission system. Superconductivity 44(5), 27-30 (2016)

18. AP Sample, DA Meyer, JR Smith, Analysis, experimental results, and range adaptation of magnetically coupled resonators for wireless power transfer IEEE Trans. Ind. Electron. 58(2), 544-554 (2011)

19. A Karalis, JD Joannopoulos, M Soljačić, Efficient wireless non-radiative mid-range energy transfer. Ann. Phys. 323(1), 34-48 (2008)

\section{Submit your manuscript to a SpringerOpen ${ }^{\circ}$ journal and benefit from:}

- Convenient online submission

- Rigorous peer review

- Open access: articles freely available online

- High visibility within the field

- Retaining the copyright to your article

Submit your next manuscript at springeropen.com 